\title{
Potencial inseticida de óleos essenciais sobre Tribolium castaneum em milho armazenado
}

\author{
MAGALHÃES, C.R.I. ${ }^{*} ;$ OLIVEIRA, C.R.F."; MATOS, C.H.C.'; BRITO, S.S.S.2; MAGALHÃES, T.A.'; FERRAZ, \\ M.S.S. ${ }^{1}$ \\ 'Universidade Federal Rural de Pernambuco - Programa de Pós-graduação em Produção Vegetal, Unidade \\ Acadêmica de Serra Talhada, Avenida Gregório Ferraz Nogueira, S/N, José Tomé de Souza Ramos, CEP: \\ 56909-535; 'Universidade Federal Rural de Pernambuco - Programa de Pós-graduação em Produção Agrícola, \\ Unidade Acadêmica de Garanhuns, Avenida Bom Pastor, S/N, Boa Vista, CEP: 55292-270, Garanhuns - PE. \\ *Autor para correspondência: cilenerejane@hotmail.com
}

RESUMO: O presente trabalho teve como objetivo investigar o potencial inseticida de óleos essenciais de Croton heliotropiifolius, Croton pulegiodorus, Myracrodruon urundeuva e Ocimum basilicum sobre adultos de Tribolium castaneum em milho armazenado. Para cada óleo foram realizados bioensaios de fumigação, repelência e o efeito sobre a taxa instantânea de crescimento $\left(r_{i}\right)$, em cinco concentrações $(0 ; 5 ; 10 ; 15$ e $20 \mu \mathrm{L})$. Os bioensaios foram conduzidos sob condições constantes de temperatura $\left(28 \pm 2{ }^{\circ} \mathrm{C}\right)$, umidade relativa $(70 \pm 5 \%)$ e escoto fase de 24 horas. Nos testes de fumigação diferentes concentrações dos óleos foi aplicado em tiras de papel filtro presas na parte inferior da tampa da câmara de fumigação $(1,5 \mathrm{~L})$, a qual continha 20 gramas de substrato alimentar e 10 insetos adultos de $T$. castaneum não sexados. A mortalidade dos insetos foi avaliada após 48 horas de exposição. Os testes de repelência foram efetuados em arenas compostas por dois frascos ligados a uma caixa central. Em um frasco foi depositado o substrato alimentar com diferentes concentrações do óleo essencial, e, no outro, foi depositado apenas alimento (testemunha). Dez insetos adultos foram liberados na caixa central, ficando expostos por 5 dias para avaliação da preferência. Nos bioensaios de fumigação observou-se atividade inseticida do óleo essencial de $M$. urundeuva sobre adultos de T. castaneum. Nos bioensaios de repelência, todos os óleos testados apresentaram efeito repelente. A emergência de T. castaneum reduziu entre 33 e 100\% quando foram criados em pó de milho tratado com os óleos essenciais. Os óleos essenciais de C. pulegiodorus e $O$. basilicum ocasionaram redução do crescimento populacional de $T$. castaneum em grãos de milho tratados. Os óleos testados demonstraram ser uma alternativa eficiente de controle para o uso nos programas de manejo de $T$. castaneum em unidades armazenadoras.

Palavras-chave: Controle alternativo, inseticidas botânicos, grãos armazenados.

\begin{abstract}
Insecticidal potential of essential oils on Tribolium castaneum in stored maize. The current work aimed at to investigate the insecticide potential of essential oils of Croton heliotropiifolius, Croton pulegiodorus, Myracrodruon urundeuva and Ocimum basilicum on adults of Tribolium castaneum in stored maize. For each oil, it were performed fumigation tests, repellency and the effect on the instantaneous rate of increase $\left(r_{i}\right)$, in five concentrations $(0 ; 5 ; 10 ; 15$ e $20 \mu \mathrm{L})$. Bioassays were carried out under constant temperature conditions $(28 \pm 2$ $\left.{ }^{\circ} \mathrm{C}\right)$, relative humidity $(70 \pm 5 \%)$ and scot phase of 24 hours. In fumigation different concentrations of test oils were applied on filter paper strips attached on the bottom of the fumigation chamber cover $(1.5 \mathrm{~L})$, which contained 20 grams of food substrate and 10 unsexed adults of $T$. castaneum. The insect mortality was recorded after 48 hours of exposure. The repellency tests were performed in arenas composed of two pots connected to a central box. In a pot it was deposited feed substrate, with concentrations of the essential oil, in the other pot (control) it was deposited only food. Ten adult insects were released in the central box, being exposed for 5 days to evaluate the preference. In the fumigation tests, insecticide activity of the essential oil of M. urundeuva was observed on adults of $T$. castaneum. In the repellency tests, all the tested oils presented repellent effect. The emergency of $T$. castaneum reduced between $33 \%$ and $100 \%$ when they were in the powdered maize treated with the essential oils. Maize grains
\end{abstract}


treated with $C$. pulegiodorus and $O$. basilicum essential oils caused a significant decrease in the populations of $T$. castaneum. The tested oils proved to be an efficient control alternative for the use in managing programs of $T$. castaneum in storing units.

Key words: Alternative control, botanical insecticides, stored grains.

\section{INTRODUÇÃO}

O Tribolium castaneum (Herbst, 1797) (Coleoptera: Tenebrionidae) é considerado uma praga secundária e cosmopolita (Pacheco \& Paula, 1995) e, geralmente, sua presença é sinal de que os grãos estão infestados por pragas primárias. Se as condições de armazenamento forem favoráveis ao seu desenvolvimento, $T$. castaneum pode causar prejuízos ainda maiores aos causados pelo ataque das pragas que permitiram sua instalação (Trematerra, 2000). Ele desenvolve melhor em massas de grãos com alto teor de impurezas e grãos quebrados, danificados pelo manuseio mecanizado durante os processos de colheita, secagem e armazenamento ou, ainda, pode valerse dos orifícios deixados pelos insetos primários, como os gorgulhos (Sokoloff, 1974).

A utilização de produtos químicos de diferentes classes toxicológicas é o método de controle mais utilizado contra pragas de armazenamento, incluindo o T. castaneum. Apesar da eficiência que esses produtos possuem, o uso intensivo pode acarretar vários problemas como o acúmulo de resíduos tóxicos nos alimentos de consumo humano, contaminação do ambiente e surgimento de resistência entre os insetos, dentre outros (Faroni et al., 1995).

Recentemente várias pesquisas têm sido direcionadas à bioatividade de óleos essenciais de plantas ou seus constituintes químicos como possível alternativa para o uso de inseticidas sintéticos (Rajendran \& Srianjini, 2008). Neste sentido, muitos estudos vêm demonstrando que os óleos essenciais tem potencial promissor como fumigante sobre vários insetos-praga de produtos armazenados, incluindo $T$. castaneum (Clemente et al., 2003; Mondal \& Khalequzzaman, 2006; Chu et al., 2011; Nattudurai et al., 2012).

Foi demonstrado que os produtos extraídos de plantas (óleos essenciais ou seus componentes voláteis, por exemplo) apresentam atividade ovicida, inseticida, repelente, atraente, ou podem servir como deterrentes para oviposição ou alimentação, e ainda, inibir o crescimento dos insetos, dentre outros (Tunc et al., 2000; Lee et al., 2004; Isman, 2006 Negahban \& Moharramipour, 2007). E a toxicidade dos óleos essenciais pode ocorrer por contato, ingestão ou fumigação (Rajendran \& Srianjini, 2008 ).

Diante da importância econômica de
T. castaneum, devido aos grandes prejuízos causados por essa praga e da necessidade de se elaborar métodos e instrumentos que possibilitem o manejo ecológico de insetos-praga, através da bioatividade de óleos vegetais, o presente trabalho teve como objetivo investigar o potencial inseticida de óleos essenciais de Croton heliotropiifolius Kunth (Euphorbiaceae), Croton pulegiodorus Baill (Euphorbiaceae), Myracrodruon urundeuva Allemão (Anacardiaceae) e Ocimum basilicum Linnaeus sobre adultos de $T$. castaneum em pó de milho armazenado.

\section{MATERIAL E MÉTODOS}

O trabalho foi desenvolvido nos Laboratórios de Entomologia e de Biologia da Unidade Acadêmica de Serra Talhada (UAST/UFRPE), conduzidos em câmara climatizada ajustada a $28 \pm 2{ }^{\circ} \mathrm{C}, 70 \pm 5 \%$ UR e fotofase de 24 horas.

\section{Criação de Tribolium castaneum}

Os insetos foram mantidos em frascos de vidro de 1,5 L, fechados com tampa plástica perfurada e revestida internamente com tecido fino que permitisse as trocas gasosas. As criações foram mantidas em câmara climática tipo B.O.D, a $28 \pm 2$ ${ }^{\circ} \mathrm{C}, 70 \pm 5 \%$ de UR e $24 \mathrm{~h}$ de fotofase, sendo usado como substrato alimentar pó de milho, com teor de umidade de $13 \%$ base úmida (b.u.).

Os insetos foram confinados durante 15 dias para efetuarem a postura, em seguida foram retirados e os recipientes estocados até a emergência da geração seguinte. Este procedimento foi realizado por várias vezes, para garantir a quantidade de adultos necessários para a execução de todos os experimentos.

\section{Óleos essenciais}

Os óleos essenciais de C. heliotropiifolius, C. pulegiodorus, $M$. urundeuva foram extraídos das folhas pelo processo de hidrodestilação, através do equipamento tipo Clevenger modificado, utilizandose $200 \mathrm{~g}$ em $3 \mathrm{~L}$ de água destilada por duas horas. As frações obtidas foram separadas da água por diclorometano e secas com sulfato de sódio anidro $\left(\mathrm{Na}_{2} \mathrm{SO}_{4}\right)$, sendo levadas posteriormente ao rotaevaporador, onde foi retirado o diclorometano, obtendo-se então o óleo essencial. 
O material botânico referente às espécies utilizadas está depositado no Herbário do Semiárido do Brasil (HESBRA) da UFRPE (Tabela 1). O óleo essencial de manjericão (Ocimum basilicum) foi adquirido da empresa Florananda Ind. e Com. de cosméticos e produtos naturais LTDA.

\section{Eliminação da infestação e equilíbrio da umidade do pó do milho \\ O pó de milho utilizado para criações} e montagem de experimentos foi obtido no Mercado Público de Serra Talhada-PE. O pó foi acondicionado em sacos plásticos e mantido em freezer sob temperatura de $-10{ }^{\circ} \mathrm{C}$, durante três dias. Após a retirada do freezer, foi transferido para frascos de vidro, mantido no laboratório à temperatura ambiente.

\section{Efeito fumigante de óleos essenciais sobre Tribolium castaneum}

Foram utilizadas como câmaras de fumigação, recipientes de vidro (tipo bomboniere) de $1,5 \mathrm{~L}$ de capacidade, onde foram confinados 10 adultos de $T$. castaneum não sexados e com até 15 dias de idade, de acordo com a metodologia adaptada de Aslan et al. (2004). Os óleos foram impregnados com pipetador automático, em tiras de papel de filtro de $5 \times 2 \mathrm{~cm}$, fixadas na superfície inferior da tampa dos recipientes, com as seguintes concentrações: 0; 5; 10; 15 e $20 \mu \mathrm{L} / \mathrm{L}$, em $20 \mathrm{~g}$ de milho. Estas concentrações foram selecionadas com base em Keita et al. (2001) e Brito (2014).

Decorridas 48 horas da montagem dos experimentos, avaliou-se a porcentagem de mortalidade. Foram efetuados experimentos individuais para cada óleo essencial, em delineamento inteiramente casualizado, em quatro repetições (Santos, 2009). Os resultados foram submetidos à análise de regressão, utilizando-se o programa computacional ASSISTAT versão 7.7 Beta (Silva, 2013).

\section{Efeito repelente de óleos essenciais sobre Tribolium castaneum}

Os óleos foram testados em arenas compostas de dois recipientes plásticos, interligados simetricamente a um pote central por dois tubos plásticos. Em um dos potes colocou-se $20 \mathrm{~g}$ de pó de milho tratadas com o óleo essencial e no outro a mesma quantidade de pó não tratado (testemunha). Os óleos foram aplicados diretamente no pó de milho com pipetador automático, tendo sido o pó homogeneizado por 30 segundos. No pote central foram liberados 10 adultos não sexados de $T$. castaneum com até 15 dias de idade.

Após cinco dias da liberação no pote central, os insetos contidos em cada recipiente foram contados para avaliação da repelência. Os insetos foram descartados e o pó acondicionado em outros recipientes plásticos. Para todos os óleos testados, foi quantificado o número de insetos atraídos ou repelidos. Além disso, foi contabilizado o número de adultos emergidos (Gusmão, 2012), após 22 dias da infestação, que foi utilizado para o cálculo do percentual de redução de emergência de adultos.

$O$ índice de repelência (IR) foi avaliado pela fórmula: $I R=2 G /(G+P)$, onde $G=\%$ de insetos atraídos no tratamento e $P=\%$ de atraídos na testemunha. O intervalo de segurança (IS) utilizado para considerar se o tratamento é ou não repelente foi obtido, usando-se a média dos IR (índice de repelência) e o respectivo desvio padrão (DP), ou seja, se a média dos IR for menor que 1 - DP, o óleo é repelente; se for maior que 1 + DP o óleo é atraente e se estiver entre 1 - DP e

1 + DP o óleo é considerado neutro. Este índice é uma adaptação da fórmula citada por Lin et al. (1990), para índice de consumo.

O percentual médio de emergência de adultos foi calculado de acordo com a seguinte fórmula: $\mathrm{PR}=[(\mathrm{NC}-\mathrm{NT}) /(\mathrm{NC}+\mathrm{NT}) \times 100]$, sendo $\mathrm{PR}=$ percentual médio de emergência, $\mathrm{NC}=$ média de insetos emergidos na testemunha e NT= média de insetos emergidos no tratamento (Obeng-Ofori, 1995).

Cada óleo essencial foi testado nas diferentes concentrações utilizadas $(0 ; 5 ; 10 ; 15$ e 20 $\mu \mathrm{L} / \mathrm{L})$, separadamente com a respectiva testemunha, em delineamento inteiramente casualizado com quatro repetições (Santos, 2009). Os resultados foram submetidos à análise de variância e as médias comparadas pelo teste $\mathrm{t}(P<0,05)$ (Oliveira \& Vendramim, 1999), utilizando-se o programa computacional ASSISTAT 7.7 versão Beta (Silva, 2013).

TABELA 1. Plantas coletadas para estudo do efeito dos óleos essenciais em T. castaneum.

\begin{tabular}{lllll}
\hline Nome Científico & Família & Nome vulgar & Voucher & Local de coleta \\
\hline Croton heliotropiifolius & Euphorbiaceae & Velame & S.S. Matos 109 & Triunfo-PE \\
Croton pulegiodorus & Euphorbiaceae & Velaminho & S.S. Matos 104 & Triunfo-PE \\
Myracrodruon urundeuva & Anacardiaceae & Aroeira-do-sertão & S.S. Matos 455 & Serra Talhada - PE \\
\hline
\end{tabular}

Rev. Bras. PI. Med., Campinas, v.17, n.4, supl. III, p.1150-1158, 2015. 


\section{Efeito de óleos essenciais sobre a taxa instantânea de crescimento (ri) de Tribolium castaneum}

Neste experimento as unidades experimentais consistiram de potes fechados com tampa plástica perfurada e revestida internamente com tecido fino para permitir as trocas gasosas, contendo $20 \mathrm{~g}$ de pó de milho. Os óleos foram aplicados diretamente sobre o pó de milho com pipetador automático, tendo sido o pó homogeneizado por 30 segundos. Em cada pote foram liberados 10 adultos não sexados de $T$. castaneum com até 15 dias de idade. As unidades experimentais foram armazenadas por 60 dias em câmara climatizada ajustada a $28 \pm 2{ }^{\circ} \mathrm{C}, 60 \pm 5 \%$ UR e fotofase de 24 horas.

Após os 60 dias, foram avaliados o número de insetos emergidos e calculada a taxa instantânea de crescimento populacional. Para este cálculo utilizou-se a equação: $r_{i}=[\ln (\mathrm{Nf} / \mathrm{N} 0) / \Delta \mathrm{T}]$, onde $\mathrm{Nf}$ = Número final de insetos; N0 = Número inicial de insetos e $\Delta T=$ Variação de tempo (número de dias em que o ensaio foi executado). Valor positivo de $r_{i}$ indica crescimento populacional; $r_{i}=0$ significa que a população encontra-se estável e valor negativo de $r_{i}$ indica declínio da população até a extinção, quando $\mathrm{Nf}=0$ (Walthall \& Stark, 1997).

Cada óleo essencial foi testado separadamente, em delineamento inteiramente casualizado com cinco tratamentos $(0 ; 5 ; 10 ; 15$ e $20 \mu \mathrm{L} \mathrm{L}^{-1}$ ) e quatro repetições. Os resultados foram submetidos à analise de variância e as médias comparadas pelo teste Tukey a $5 \%$ de probabilidade, utilizando-se o programa computacional ASSISTAT versão 7.7 Beta (Silva, 2013).

\section{RESULTADOS E DISCUSSÃO \\ Efeito fumigante de óleos essenciais sobre Tribolium castaneum}

Com relação ao efeito fumigante, não foram observadas diferenças significativas para as concentrações testadas dos óleos essenciais de C. heliotropiifolius, C. pulegiodorus e $O$. basilicum sobre T. castaneum. Por outro lado, observou-se que o óleo essencial de $M$. urundeuva ocasionou $12,5 \%$ de mortalidade dos adultos de $T$. castaneum, sendo que a mortalidade aumentou com o aumento das concentrações testadas (Figura 1).

Para Sá et al. (2008) a defesa química de plantas envolve metabólitos secundários de baixo peso molecular assim como lectinas. Os autores demonstraram que extratos salinos do cerne de $M$. urundeuva e a lectina isolada deste tecido vegetal apresentaram atividade inseticida contra cupins.

Silva (2000) estudando o efeito do extrato de folhas de $M$. urundeuva, constatou atividade inseticida sobre $S$. zeamais, induzindo a mortalidade de modo dose-dependente, além de promover distúrbios nutricionais e de crescimento. Rajendran \& Sriranjini (2008) estudando o efeito fumigante de óleos essenciais de várias plantas, também comentam do efeito tóxico sobre adultos de $T$. castaneum, corroborando com os dados aqui apresentados. Já Fernandes (2011) constatou que os extratos etanólicos de casca e caule de $M$. urundeuva produziram um efeito antifúngico nas espécies de cândidas $C$. albicans e $C$. tropicalis. Os autores concluíram que esta espécie tem potencial biotecnológico para estudos visando à produção de um fitofármaco.

Queiroz et al. (2002) observaram a presença

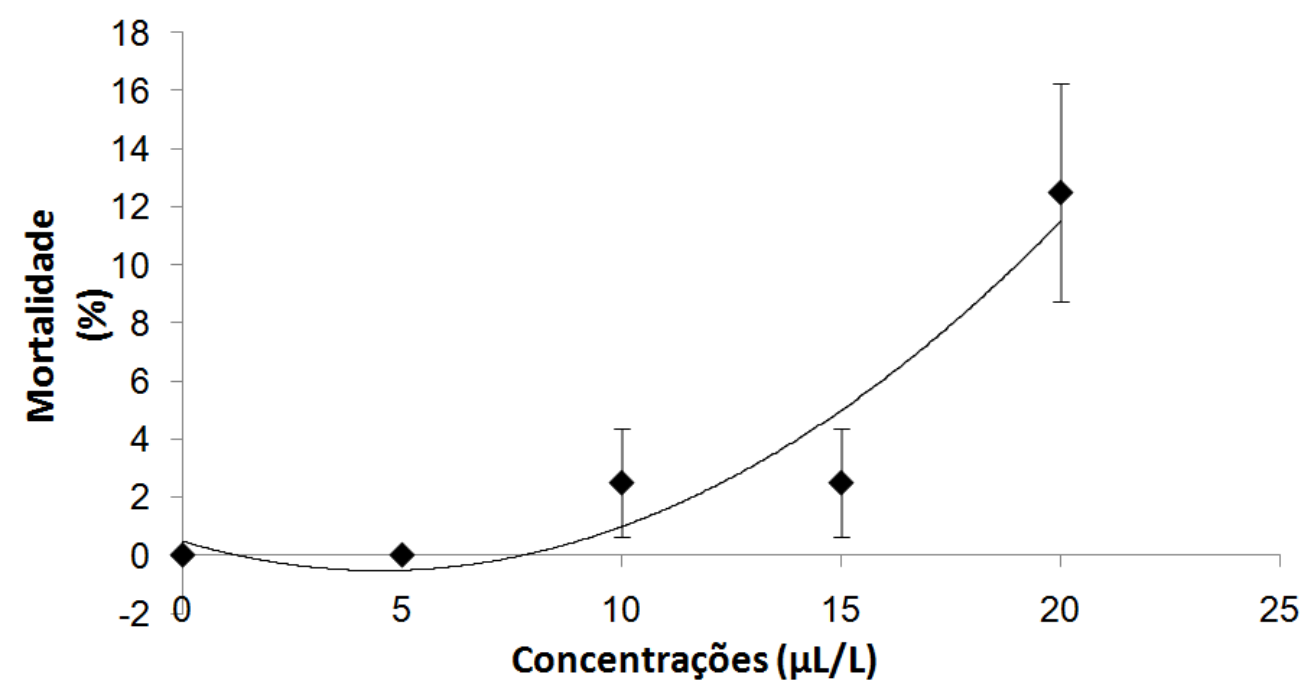

FIGURA 1. Efeito fumigante do óleo essencial de Myracrodruon urundeuva sobre adultos de Tribolium castaneum $\left(y=0,05 x^{2}-0,45 x+0,5 ; R^{2}=0,90 ; F=10,67 ; P<0,001\right)$.

Rev. Bras. PI. Med., Campinas, v.17, n.4, supl. III, p.1150-1158, 2015. 
de taninos em M. urundeuva, que são considerados redutores digestivos. Foi observado que os taninos reduzem significativamente o crescimento e a sobrevivência dos insetos, uma vez que inativam enzimas digestivas e criam um complexo de difícil digestão (Mello \& Silva-Filho, 2002). Alguns estudos tem demonstrado vários constituintes voláteis, dentre eles o $\beta$-cariofileno (Sá, 2008), que pode estar relacionado ao efeito fumigante desta espécie. A madeira de $M$. urundeuva também possui lectinas vegetais que mostraram atividade tóxica contra larvas de C. maculatus (Macedo et al., 2004).

\section{Efeito repelente de óleos essenciais sobre Tribolium castaneum \\ Observou-se que as concentrações de 5} e $15 \mu \mathrm{L} \mathrm{L}^{-1}$ do óleo essencial de C. heliotropiifolius, foram consideradas neutras e as demais, repelentes. O mesmo foi observado para o óleo de M. urundeuva. Para C. heliotropiifolius houve diferença significativa entre as concentrações de 10 e $15 \mu \mathrm{L} \mathrm{L-1}$ e suas testemunhas pelo teste " $t$ " $(P<0,05)($ Tabela 2$)$.

Por outro lado, apenas o óleo essencial de C. pulegiodorus foi considerado neutro em todas as concentrações avaliadas, havendo diferença significativa entre todas as concentrações e suas testemunhas. Em O. basilicum a concentração de
$15 \mu L^{-1}{ }^{-1}$ foi considerada repelente, sendo as demais consideradas neutras. Não foram observadas diferenças significativas entre as concentrações de $O$. basilicum e suas respectivas testemunhas (Tabela 2).

Liu \& Ho (1999) avaliaram o efeito repelente do óleo essencial de Evodia rutaecarpa Juss. (Rutaceae) e constataram que este óleo mostrou forte efeito repelente sobre $T$. castaneum, fato que também foi observado com os óleos de $C$. heliotropiifolius, C. pulegiodorus, M. urundeuva e O. basilicum neste estudo.

Pauliquevis et al. (2013) observaram maior efeito repelente do óleo essencial de $P$. umbellata sobre R. dominica na menor dose. Os autores observaram que doses altas ocasionaram falta de movimentação dos insetos, com posterior distribuição aleatória pela arena. Segundo os autores, algumas substâncias vegetais, dependendo da concentração, podem provocar efeitos fisiológicos ou comportamentais, diminuindo ou interrompendo o movimento do inseto nas concentrações mais altas. Isso pode explicar o comportamento ocasionado por algumas concentrações mais altas no presente estudo (Tabela 2).

As porcentagens de redução de adultos emergidos de $T$. castaneum variaram de 33,33 a

TABELA 2. Efeito repelente de óleos essenciais sobre adultos de T. castaneum em milho tratado. Temp.: $28 \pm$ $2^{\circ} \mathrm{C}$, UR: $70 \pm 5 \%$ e fotofase: 24 horas.

\begin{tabular}{|c|c|c|c|c|c|}
\hline \multirow[t]{2}{*}{ Tratamento } & \multirow[t]{2}{*}{$\begin{array}{l}\text { Concentração } \\
\left(\mu \mathrm{L} \mathrm{L}^{-1} / 20 \mathrm{~g}\right)\end{array}$} & \multicolumn{2}{|c|}{$\begin{array}{c}\text { Adultos Atraídos } \\
\qquad(\%)\end{array}$} & \multirow[t]{2}{*}{$\mathrm{IR} \pm \mathrm{DP}^{1}$} & \multirow[t]{2}{*}{ IS } \\
\hline & & Óleo & Testemunha & & \\
\hline \multirow{4}{*}{ C. heliotropiifolius } & $5 \mu \mathrm{L} \mathrm{L}^{-1}$ & 25,00 & 75,00 * & $0,50 \pm 0,52$ & $\mathrm{~N}$ \\
\hline & $10 \mu \mathrm{L} \mathrm{L}^{-1}$ & 20,00 & 62,50 ns & $0,45 \pm 0,66$ & $\mathrm{R}$ \\
\hline & $15 \mu L^{-1}$ & 30,00 & $60,00^{\text {ns }}$ & $0,72 \pm 0,59$ & $\mathrm{~N}$ \\
\hline & $20 \mu \mathrm{L} \mathrm{L}^{-1}$ & 30,00 & 67,50 * & $0,40 \pm 0,66$ & $\mathrm{R}$ \\
\hline \multirow{4}{*}{ C. pulegiodorus } & $5 \mu \mathrm{L} \mathrm{L}^{-1}$ & 10,00 & 70,00 * & $0,19 \pm 0,28$ & $\mathrm{~N}$ \\
\hline & $10 \mu L^{-1}$ & 12,50 & 82,50 * & $0,25 \pm 0,19$ & $\mathrm{~N}$ \\
\hline & $15 \mu \mathrm{L} \mathrm{L}^{-1}$ & 12,50 & 82,50 * & $0,25 \pm 0,50$ & $\mathrm{~N}$ \\
\hline & $20 \mu \mathrm{L} \mathrm{L}^{-1}$ & 0,250 & 97,50 * & $0,05 \pm 0,10$ & $\mathrm{~N}$ \\
\hline \multirow{4}{*}{ M. urundeuva } & $5 \mu \mathrm{L} \mathrm{L}^{-1}$ & 0,75 & 77,50 * & $0,15 \pm 0,30$ & $\mathrm{~N}$ \\
\hline & $10 \mu \mathrm{L} \mathrm{L}^{-1}$ & 15,00 & $30,00^{\text {ns }}$ & $0,34 \pm 0,40$ & $\mathrm{R}$ \\
\hline & $15 \mu L^{-1}$ & 17,50 & 50,00 * & $0,41 \pm 0,47$ & $\mathrm{~N}$ \\
\hline & $20 \mu \mathrm{L} \mathrm{L}^{-1}$ & 15,00 & 80,00 * & $0,31 \pm 0,10$ & $\mathrm{R}$ \\
\hline \multirow{4}{*}{ O. basilicum } & $5 \mu L^{-1}$ & 30,00 & $42,50 \mathrm{~ns}$ & $0,78 \pm 0,58$ & $\mathrm{~N}$ \\
\hline & $10 \mu \mathrm{L} \mathrm{L}^{-1}$ & 47,50 & 42,50 ns & $1,07 \pm 0,31$ & $\mathrm{~N}$ \\
\hline & $15 \mu L^{-1}$ & 27,50 & $17,50^{\mathrm{ns}}$ & $0,12 \pm 0,25$ & $\mathrm{R}$ \\
\hline & $20 \mu \mathrm{L} \mathrm{L}^{-1}$ & 0,75 & 0,50 ns & $1,00 \pm 1,15$ & $\mathrm{~N}$ \\
\hline
\end{tabular}

\footnotetext{
* Médias, nas linhas, diferem entre si pelo teste "t" $(P<0,1$ u $P<0,05)$.

ns Médias, nas linhas, não diferem entre si pelo teste "t" $(P<0,05)$.

1 Índice de Repelência e Desvio Padrão.

${ }^{2}$ Intervalo de Segurança, onde R= Repelente, N= Neutro e A= Atraente.
} 
$100 \%$, havendo destaque para o óleo essencial de O. basilicum nas concentrações de 5,10 e $20 \mu \mathrm{L}$ $\mathrm{L}^{-1}$ (Tabela 3). O mesmo comportamento ocorreu para os óleos essenciais de C. pulegiodorus e $C$. heliotropiifolius, na concentração de $20 \mu \mathrm{L} \mathrm{L}^{-1}$ e $M$. urundeuva na concentração de $15 \mu L^{L^{-1}}$. Neste sentido, constatou-se que houve efeito ovicida/ larvicida nestas concentrações, ocasionando redução no percentual de emergência de adultos de T. castaneum (Tabela 3 ). Além disso, como o número de adultos atraídos pelos óleos em geral foi menor, ocorreu menor taxa de oviposição e emergência.

Alguns estudos demonstraram que os óleos essenciais afetam diretamente o desenvolvimento embrionário dos ovos, reduzindo assim a emergência de novos adultos, causando efeitos ovicida/larvicida (Tapondjou et al., 2005; Ketoh et al., 2005). Segundo Luz et al. (2009) os componentes majoritários do óleo essencial de $O$. basilicum são o linalol e o geraniol. Provavelmente estes compostos apresentam alta toxidade a $T$. castaneum, provocando, assim, a sua morte. O linalol vem sendo utilizado para sintetizar diversos compostos, como o acetato de linalila, que está sendo testado como acaricida, bactericida e fungicida (Morais, 2006). Para o gênero Croton, alguns estudos de análise da composição química das espécies revelou a presença de monoterpenoides, sesquiterpenoides e fenilpropanoides como constituintes majoritários (Torres, 2008; Santana, 2011). Para M. urundeuva foram identificados 16 constituintes voláteis, sendo o sesquiterpeno $\beta$-cariofileno o principal (Sá, 2008).

Upadhyay \& Jaiswal (2007), analisando a atividade biológica do óleo essencial de Piper nigrum contra $T$. castaneum, observaram que as concentrações 0,3 e 0,4 $\mu \mathrm{l}$ repeliram 95 e 97,5\% dos insetos. Quando utilizaram $8 \mu \mathrm{l}$ a sobrevivência de larvas e pupas foi de aproximadamente $80 \%$ e a emergência de adultos foi reduzida para $20 \%$. Ko Ko et al. (2009) estudando a atividade repelente e tóxica por fumigação e contato do óleo essencial de Melaleuca cajuputi, observaram que o mesmo foi mais eficiente para repelir ( $96 \%$ em média) esse inseto na concentração de $0,63 \mu \mathrm{g} \mathrm{cm}^{-2}$.

De acordo com Coitinho et al. (2006) o efeito repelente é uma propriedade relevante na escolha de um óleo essencial para o controle de pragas de grãos armazenados, pois quanto maior a repelência, menor será a infestação, resultando na redução ou supressão da postura e, consequentemente, do número de insetos emergidos, fato que ocorreu nos experimentos realizados. Assim, o emprego de óleos essenciais poderá favorecer principalmente aos pequenos e médios produtores de milho,

TABELA 3. Porcentagem de redução do número de adultos de T. castaneum emergidos em milho tratado com óleos essenciais.

\begin{tabular}{|c|c|c|c|c|}
\hline \multirow{2}{*}{ Tratamentos } & \multirow[t]{2}{*}{ Concentração ( $\mu \mathrm{L} \mathrm{L}^{-1 / 20 \mathrm{~g})}$} & \multicolumn{2}{|c|}{ Média de Adultos Emergidos ( \pm DP) } & \multirow{2}{*}{ Redução (\%) } \\
\hline & & Óleo & Testemunha & \\
\hline \multirow{4}{*}{ C.heliotropiifolius } & $5 \mu \mathrm{L} \mathrm{L}^{-1}$ & 0,25 & 0,75 ns & 50,00 \\
\hline & $10 \mu \mathrm{L} \mathrm{L}^{-1}$ & 0,25 & 0,75 ns & 50,00 \\
\hline & $15 \mu \mathrm{L} \mathrm{L}^{-1}$ & 0,25 & $0,50 \mathrm{~ns}$ & 33,33 \\
\hline & $20 \mu \mathrm{L} \mathrm{L}^{-1}$ & 0,00 & 0,75 * & 100,00 \\
\hline \multirow{4}{*}{ C. pulegiodorus } & $5 \mu \mathrm{L} \mathrm{L}^{-1}$ & 0,25 & 0,50 ns & 33,33 \\
\hline & $10 \mu \mathrm{L} \mathrm{L}^{-1}$ & 0,25 & 0,50 ns & 33,33 \\
\hline & $15 \mu \mathrm{L} \mathrm{L}^{-1}$ & 0,75 & 0,25 ns & NF \\
\hline & $20 \mu \mathrm{L} \mathrm{L}^{-1}$ & 0,00 & 2,25 ns & 100,00 \\
\hline \multirow{4}{*}{ M. urundeuva } & $5 \mu \mathrm{L} \mathrm{L}^{-1}$ & 0,25 & 0,75 ns & 50,00 \\
\hline & $10 \mu \mathrm{L} \mathrm{L}^{-1}$ & 0,25 & $0,50^{\text {ns }}$ & 33,33 \\
\hline & $15 \mu \mathrm{L} \mathrm{L}^{-1}$ & 0,00 & 0,50 ns & 100,00 \\
\hline & $20 \mu \mathrm{L} \mathrm{L}^{-1}$ & 0,25 & $0,75^{\text {ns }}$ & 50,00 \\
\hline \multirow{4}{*}{ O. basilicum } & $5 \mu \mathrm{L} \mathrm{L}^{-1}$ & 0,00 & 0,75 ns & 100,00 \\
\hline & $10 \mu \mathrm{L} \mathrm{L}^{-1}$ & 0,00 & $0,75^{\text {ns }}$ & 100,00 \\
\hline & $15 \mu \mathrm{L} \mathrm{L}^{-1}$ & 0,25 & 0,75 ns & 50,00 \\
\hline & $20 \mu \mathrm{L} \mathrm{L}^{-1}$ & 0,00 & $0,75^{*}$ & 100,00 \\
\hline
\end{tabular}

*. Médias, nas linhas, diferem estatisticamente entre si pelo teste " $t$ " $(P<0,05)$.

ns Médias, nas linhas, não diferem entre si pelo teste " $t$ " $(P<0,05)$.

NF - não se adequou a fórmula.

DP - Desvio Padrão.

Rev. Bras. PI. Med., Campinas, v.17, n.4, supl. III, p.1150-1158, 2015. 
devido à eficácia, facilidade de manuseio e rápida degradação, contribuindo para o estabelecimento de uma agricultura sustentável (Roel, 2001; Keita et al., 2001).

\section{Efeito de óleos essenciais sobre a taxa instantânea de crescimento $\left(r_{i}\right)$ de Tribolium castaneum \\ Com relação à taxa instantânea de} crescimento de $T$. castaneum em milho tratado com os óleos essenciais, só houve efeito significativo dos óleos de C. pulegiodorus e de $O$. basilicum, os quais proporcionaram menor taxa de crescimento do inseto quando comparados à testemunha (Tabela 4).

Tais resultados demonstram que estes óleos podem ser utilizados na menor concentração testada ( $\left.5 \mu \mathrm{L} \mathrm{L}^{-1}\right)$, já que tanto para C. pulegiodorus como para $O$. basilicum, não houve diferença na taxa de crescimento entre as concentrações testadas (Tabela 4). Neste sentido, proporcionaram taxas instantâneas positivas, mas em valores inferiores quando comparadas à testemunha.

Os baixos valores observados para as taxas de crescimento $\left(r_{i}\right)$ de $T$. castaneum, submetido aos óleos testados, indicam que os mesmos podem reduzir o numero de gerações desse inseto-praga, em relação a grãos não tratados. Neste sentido, a toxicidade dos óleos essenciais pode ter atuado sobre ovos e larvas de T. castaneum, implicando na diminuição das populações deste inseto.

\section{CONCLUSÕES}

Os óleos essenciais avaliados não apresentaram efeito fumigante sobre $T$. castaneum, com exceção de $M$. urundeuva, que promoveu baixa mortalidade mesmo na maior concentração testada, caracterizando fraco potencial inseticida nestas condições.

De maneira geral os óleos essenciais de C. heliotropiifolius, $M$. urundeuva e $O$. basilicum mostraram-se repelentes ou neutros para $T$. castaneum, enquanto que C. pulegiodorus apresentou-se neutro.

Os óleos essenciais de $O$. basilicum, $C$. pulegiodorus e C. heliotropiifolius ocasionaram $100 \%$ de redução da emergência de adultos de $T$. castaneum, apresentando efeito ovicida/larvicida.

Além disso, os óleos essenciais de $C$. pulegiodorus e $O$. basilicum reduziram a taxa instantânea de crescimento $\left(r_{i}\right)$ de T. castaneum.

TABELA 4. Efeito de óleos essenciais sobre a taxa instantânea de crescimento $\left(r_{i}\right)$ de Tribolium castaneum.

\begin{tabular}{|c|c|c|}
\hline Tratamento & 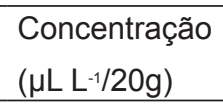 & $r_{i}$ (média) \\
\hline \multirow{5}{*}{ C. heliotropiifolius } & Testemunha & $0,00 a$ \\
\hline & $5 \mu \mathrm{LL}^{-1}$ & $-0,01^{a}$ \\
\hline & $10 \mu \mathrm{L} \mathrm{L}^{-1}$ & $-0,01 a$ \\
\hline & $15 \mu \mathrm{L} \mathrm{L}^{-1}$ & $-0,01^{a}$ \\
\hline & $20 \mu \mathrm{L} \mathrm{L}^{-1}$ & $-0,02 a$ \\
\hline \multirow{5}{*}{ C. pulegiodorus } & Testemunha & $0,04 a$ \\
\hline & $5 \mu \mathrm{L} \mathrm{L}^{-1}$ & $0,03 b$ \\
\hline & $10 \mu \mathrm{L} \mathrm{L}^{-1}$ & $0,02 b$ \\
\hline & $15 \mu \mathrm{L} \mathrm{L}^{-1}$ & $0,02 b$ \\
\hline & $20 \mu \mathrm{L} L-1$ & $0,01 \mathrm{~b}$ \\
\hline \multirow{5}{*}{ M. urundeuva } & Testemunha & $0,02^{\mathrm{a}}$ \\
\hline & $5 \mu \mathrm{L} \mathrm{L}^{-1}$ & $0,02 a$ \\
\hline & $10 \mu \mathrm{L} \mathrm{L}^{-1}$ & $0,01^{a}$ \\
\hline & $15 \mu \mathrm{L} \mathrm{L}^{-1}$ & $0,01^{a}$ \\
\hline & $20 \mu \mathrm{L} \mathrm{L}^{-1}$ & $0,01^{a}$ \\
\hline \multirow{5}{*}{ O. basilicum } & Testemunha & $0,03^{a}$ \\
\hline & $5 \mu \mathrm{LL}^{-1}$ & $0,01 b$ \\
\hline & $10 \mu \mathrm{L} \mathrm{L}^{-1}$ & $0,01 \mathrm{~b}$ \\
\hline & $15 \mu \mathrm{L} \mathrm{L}^{-1}$ & $0,01 \mathrm{~b}$ \\
\hline & $20 \mu \mathrm{L} \mathrm{L}^{-1}$ & $0,01 b$ \\
\hline
\end{tabular}

*Médias seguidas de mesma letra nas colunas não diferem estatisticamente entre si pelo teste de Tukey $(P<0,05)$. 


\section{REFERÊNCIAS}

ASLAN, I. et al. Toxicity of essential oil vapours to two greenhouse pests, Tetranychus urticae Koch and Bemisia tabaci Genn. Industrial Crops and Products, v.19, n.2, p.167-173, 2004.

BRITO, S. S. S. Manejo de coleópteros-praga de feijão armazenado com óleos essenciais. 2014. 99f. Dissertação (Mestrado - Área de Concentração em Produção Agrícola) - Departamento de Produção Agrícola, Universidade Federal Rural de Pernambuco, Garanhuns.

CHU, S. S. et al. Composition of essential oil of Chinese Chenopodium ambrosioides and insecticidal activity against maize weevil: Sitophilus zeamais. Pest Management Science, v.67, p. 714 - 718, 2011.

CLEMENTE, S. et al. Insecticidal effects of Lamiaceae species against stored products insects. Boletin de Sanidad Vegetal de Plagas, v.29, p.421-426, 2003.

COITINHO, R. L. B. C. et al. Atividade inseticida de óleos vegetais sobre Sitophilus zeamais Mots. (Coleoptera: Curculionidae) em milho armazenado. Revista Caatinga, v.19, p.176-182, 2006.

FARONI, L. R. A. et al. Utilização de produtos naturais no controle de Acanthoscelides obtectus em feijão armazenado. Revista Brasileira de Armazenamento, v.20, n.1-2, p.44-48, 1995.

FERNANDES, A. F. C. Avaliação da atividade antimicrobiana do extrato etanólico e fases particionadas de Myracrodruon urundeuva Fr. All. (Aroeira-do-sertão). 2011. 50f. Trabalho de Conclusão de Curso (Graduação - Área de Concentração em Farmácia) - Departamento de Farmácia, Universidade Estadual da Paraíba, Campina Grande.

GUSMÃO, N. M. S. Manejo de Callosobruchus maculatus (FABR.) (Coleoptera: Chrysomelidae, Bruchinae), em grãos de caupi, Vigna unguiculata (L.) Walp. com óleos essenciais. 2012. 38f. Dissertação (Mestrado - Área de Concentração em Entomologia Agrícola) - Departamento de Entomologia Agrícola, Universidade Federal Rural de Pernambuco, Recife.

ISMAN, M. B. Botanical insecticides, deterrents, and repellents in modern agriculture and an increasingly regulated world. Annual Review of Entomology, v.51, p.45-66, 2006.

KEITA, S. M. et al. Efficacy of essential oils of Ocimum basilicum $\mathrm{L}$. and $\mathrm{O}$. gratissimum $\mathrm{L}$. applied as an insecticidal fumigant and powder to control Callosobruchus maculatus (Fab.) (Coleoptera: Bruchidae). Journal of Stored Products Research, v.37, n.2, p.:339-349, 2001.

KETOH, G. K, et al., Inhibition of Callosobruchus maculatus (F.) (Coleoptera: Bruchidae) development with essential oil extracted from Cymbopogon schoenanthus L. Spreng. (Poaceae), and the wasp Dinarmus basalis (Rondani) (Hymenoptera: Pteromalidae). Journal of Stored Products Research, v.41, n.4, p.363-371, 2005

KO KO, JUNTARAJUMNONG, W.; CHANDRAPATYA, A. Repellency, fumigant and contact toxicities of Melaleuca cajuputi Powell against Sitophilus zeamais Motschulsky and Tribolium castaneum Herbst. Thai Journal of Agricultural Science, v.42, n.1, p.27-33, 2009.
LEE, B. H. et al. Fumigant toxicity of essential oils from the Myrtaceae family and 1,8-cineole. against 3 major stored-grain insects. Journal of Stored Products Research, v.40, p. 553-564, 2004.

LIN, H.; KOGAN, M.; FISHER, D. Induced resistance in soybean to the Mexican bean beetle (Coleoptera: Coccinellidae): comparisons of inducing factors. Environmental Entomology, College Park, v.19, n.6, p.1852-1857, 1990.

LIU, Z. L.; HO, S. H. Bioactivity of the essencial oil extracted from Evodia rutaecarpa Hook f. et Thomas against the grain storage insects, Sitophilus zeamais Motsch. and Tribolium castaneum (Herbst). Journal of Stored Products Research, v. 35, p.317-328, 1999.

LUZ, J. M. Q. et al. Teor, rendimento e composição química do óleo essencial de manjericão sob doses de cama de frango. Horticultura Brasileira, v.27, n.3, p.349- 353, 2009.

MACEDO, M. L. R. et al., Mechanisms of the insecticidal action of TEL (Talisia esculenta lectin) against Callosobruchus maculatus (Coleoptera: Bruchidae). Archives of Insect Biochemistry and Physiology. n.56, p.84-96, 2004.

MELLO, M. O.; SILVA-FILHO, M. C. Plant-insect interactions: an evolutionary arms race between two distinct defense mechanisms. Brazilian Journal of Plant Physiology, v.14, p.71-81, 2002.

MONDAL, M.; KHALEQUZZAMAN, M. Toxicity of essential oils against red flour beetle, Tribolium castaneum (Herbst) (Coleoptera: Tenebrionidae). Journal of Biosciences, v.14, p. 43-48, 2006.

MORAIS, T. P. S. Produção e composição do óleo essencial de manjericão (Ocimum basilicum L.) sob doses de cama de frango. 2006. 38f. Dissertação (Mestrado - Área de Concentração em Fitotecnia) Departamento de Fitotecnia, Universidade Federal de Uberlândia, Uberlândia.

NATTUDURAI, G. et al., Fumigant toxicity of volatile synthetic compounds and natural oils against red flour beetle Tribolium castaneum (Herbst) (Coleoptera: Tenebrionidae). Journal of King Saud University Science, v. 24, p.153-159, 2012.

OBENG-OFORI, D. Plant oils as grain protectants against infestations of Cryptolestes pussilus and Rhyzopertha dominica in stored grain. Entomologia Experimentalis et Applicata, v.77, n.2, p.133-139, 1995.

NEGAHBAN, M.; MOHARRAMIPOUR, S. Fumigant toxicity of Eucalyptus intertexta, Eucalyptus sargentii and Eucalyptus camaldulensis against stored product beetles. Journal of Applied Entomology, v. 131, p. 256-261, 2007.

OLIVEIRA, J. V.; VENDRAMIM, J. D. Repelência de óleos essenciais e pós vegetais sobre adultos de Zabrotes subfasciatus (Boh.) (Coleoptera: Bruchidae) em sementes de feijoeiro. Anais da Sociedade Entomológica do Brasil, Londrina, v. 28, n. 3, p. 549555, abr./set. 1999.

PACHECO, I. A.; PAULA, D. C. Insetos de grãos armazenados: identificação e biologia. $1^{\mathrm{a}}$ ed. Campinas, Fundação Cargill, 1995. 228p.

PAULIQUEVIS, C. F, et al., Atividade insetistática do óleo essencial de Pothomorphe umbellata (L.) Miq. Sobre Rhyzopertha dominica (Fabricius, 1792) (Coleoptera:

Rev. Bras. PI. Med., Campinas, v.17, n.4, supl. III, p.1150-1158, 2015. 
Bostrichidae). Revista Brasileira de Agroecologia, p.8, n.3, p. 39-45, 2013.

QUEIROZ, C. R. A. et al., Caracterização dos taninos da aroeira-preta (Myracrodruon urundeuva). Revista Árvore, v.26, p.485-492, 2002.

RAJENDRAN, S.; SRIRANJINI, V. Plant products as fumigants for stored-product insect control. Journal of Stored Products Research, v.44, p.126-135, 2008.

ROEL, A. R. Utilização de plantas com propriedades inseticidas: uma contribuição para o desenvolvimento rural sustentável. Revista Internacional de Desenvolvimento Local, v.1, n.2, p.43-50, 2001.

SÁ, R. A. Constituintes químicos da madeira-delei Myracrodruon urundeuva com propriedades antioxidantes e ação contra fungos, bactérias e insetos. 2008. 173f. Tese (Doutorado - Área de Concentração em Química Fundamental) Departamento de Química Fundamental, Universidade Federal de Pernambuco, Recife.

SANTANA, V. S. Estudo comparativo de óleos essenciais de espécies de Croton do estado de Sergipe. 2011. 95f. Dissertação (Mestrado - Área de Concentração em Química) - Departamento de Química, Universidade Federal de Sergipe, São Cristovão.

SANTOS, J. C. Susceptibilidade de Tribolium castaneum ao óleo essencial de mostarda e perspectivas de manejo de resistência. 2009. 43f. Dissertação (Mestrado - Área de Concentração em Entomologia) - Departamento de Entomologia, Universidade Federal de Viçosa, Viçosa.

SILVA, F. A. S. Assistat 7.7. UFCG, Campina Grande, 2013.
SILVA, F. A. M. et al. Efeito do estresse salino sobre a nutrição mineral e o crescimento de mudas de aroeira (Myracrodruon urundeuva) cultivadas em solução nutritiva. Revista Cerne, v.6, n.1, p.052-059, 2000.

SOKOLOFF, A. The biology of Tribolium. $1^{\text {a }}$ ed. Oxford: Oxford University Press, v.2. 1974. 610p.

TAPONDJOU, L. A. et al., Bioactivities of cymol and essential oils of Cupressus sempervirens and Eucalyptus saligna against Sitophilus zeamais Motschulsky and Tribolium confusum du Val. Journal of Stored Products Research, v. 41, p.91-102, 2005.

TREMATERRA, P. et al., Behavioural responses of Oryzaephilus surinamensis, Tribolium castaneum and Tribolium confusum to naturally and artificially damaged durum wheat kernels. Entomologia Experimentalis et Applicata, v. 94, p.195-200, 2000.

TORRES, M. C. M. Estudo químico e biológico de Croton regelianus var. matosii (Euphorbiaceae). 2008. 179f. Dissertação (Mestrado - Área de Concentração em Química Orgânica) - Departamento de Química Orgânica e Inorgânica, Universidade Federal do Ceará, Fortaleza.

TUNC, I. et al. Ovicidal activity of essential oils from five plants against two stored-product insects. Journal of Stored Products Research, v.36, p. 161-168, 2000.

UPADHYAY, R. K.; JAISWAL, G. Evaluation of biological activities of Piper nigrum oil against Tribolium castaneum. Bulletin of Insectology, v.60, n.1, p.57-61, 2007.

WALTHALL, W. K.; STARK, J. D. A comparison of acute mortality and population growth rate as endpoints of toxicological effect. Ecotoxicology and Environmental Safety, v.37, p.45-52, 1997. 